\title{
STATISTICAL CHARACTERIZATION OF
}

RICIAN MULTIPATH EFFECTS IN A MOBILE-TO-MOBILE COMMUNICATION CHANNEL

This text develops a statistical model for a narrowband mobile-to-mobile channel taking into consideration Rician scattering near receiving and transmitting antennas both individually and concomitantly. From the proposed channel model we obtain the probability density function of the received signal envelope, the time correlation function and RF spectrum of the received signal, and level crossing rates and average fade durations. We discuss the impact of these parameters on communication networks supporting an Intelligent Vehicle Highway System (IVHS). 


\subsection{Introduction}

The application of microwave data links in a land-to-mobile environment has been shown to suffer from multipath fading, shadowing, and Doppler phase shifts. These effects limit the performance of the system. It is thus desirable to have a model of the channel and its limiting effects. This text presents a statistical model for the effects of multipath fading in a mobile-to-mobile environment, extending the statistical model for Rayleigh fading by Clarke [1] and Jakes [4] for mobile-to-land, and by Akki and Haber [7] for mobile-to-mobile communication.

In a mobile channel, energy arrives at the receiver by scattering and diffraction over and/or around the surrounding environment. A short range mobile-to-mobile channel in a highway environment will also contain a much stronger direct line-of-sight component, possibly also with a strong ground reflected wave. These components combine vectorially at the receiver and give rise to a resultant signal that varies greatly depending on the distribution of the phases of the various components. These short-term variations in the received signal are called multipath fading. Long term variations in the signal, such as shadowing or path loss, are also present. The relative motion of the vehicles will give rise to a Doppler shift in the signal. Thus, the mobile radio signal varies rapidly over short distances (fading), with a local mean power that is constant over a small area, but varies slowly as the receiver moves. We will concentrate on the short term effects for narrowband channels. In contrast to [1], [4] and [7], we include a dominant component, resulting in Rician fading.

\subsection{Probability Density Function of Received Signa}

In deriving the probability density function of the received envelope, we will follow Clarke's two dimensional scattering model [1]. Work has been done by Aulin [11] to extend this to a three dimensional model [9]. However from Aulin's results it is quite clear that those waves which make a major contribution to the received signal travel in an approximately horizontal direction. We will thus continue with Clarke's model which assumes that the field incident on the mobile antenna is comprised of horizontally travelling plane waves of random phase. Also all reflections occur in a plane and both mobile are at the same height. We will augment Clarke's model by considering a dominant, 
e.g. line-of-sight, component as well as reflections at both transmitter and receiver. On a highway, reflections may also occur against vehicles in between the transmitter and receiver. However, their signal strength is often much smaller, as the path contains two relatively long segments, each with substantial free space loss. This is similar to the situation in land-to-mobile channels.

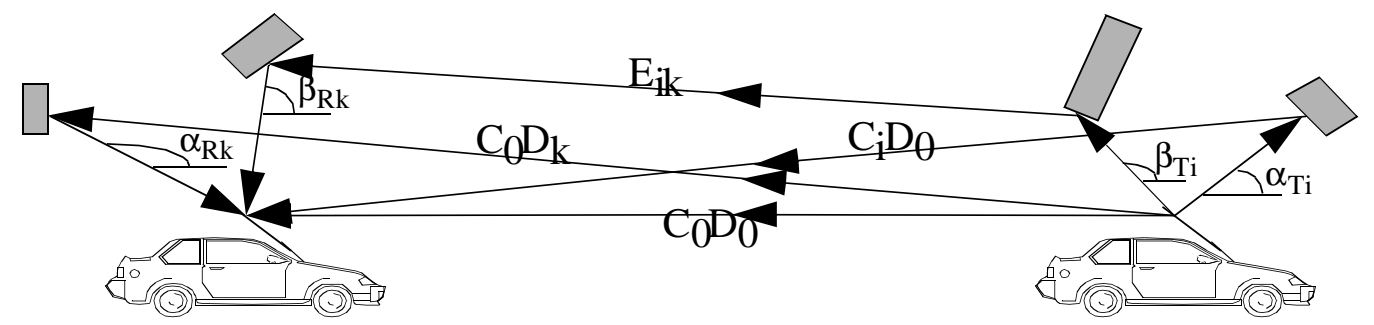

Receiver

Transmitter

Figure 2.1 Mobile-to-Mobile propagation channel with scatters near both antennas.

At every receiving point we assume the signal to be comprised of many plane waves, as shown in Fig. 2.1. Here $N_{T}$ waves experience reflections at the transmitter only, $N_{R}$ waves experience reflections at the receiver only and $N_{T} N_{R}$ waves experience reflections at both transmitter and receiver. We denote waves by an index $i$ indicating the path and reflection near the transmitter and an index $k$ denoting the path and reflections near the receiver. The $(i, k)^{\text {th }}$ incoming wave has a phase shift $\phi_{i, k}$, a spatial angle of arrival $\alpha_{R k}$, and a spatial angle of departure $\alpha_{T i}$ with respect to the velocity of the receiver. We use $i=0$ and $k=0$ for dominant waves that are not subject to scattering. The $(i, k)^{t h}$ wave has a real amplitude given by $E_{i, k}$ depending on the reflections and additional path loss that the wave undergoes. In practice the amplitudes $E_{i, k}$ may be difficult to estimate. We model this as $E_{i, k}=$ $E_{0} C_{i, k} D_{i, k}$ where $C_{i, k}$ accounts for scattering near the transmitter and $D_{i, k}$ describes scattering near the transmitter. Here $E_{0} C_{0,0} D_{0,0}$ is the deterministic amplitude of the dominant component, which in case it consists only of the line-of-sight wave, is found from free space loss. The parameters $\phi_{i, k}, \alpha_{T i}, \alpha_{R k}$, $C_{i, k}$ and $D_{i, k}$ are all assumed to be random and statistically independent, which is reasonable for a sufficiently large separation distance between transmitter and receiver. Maffett [2] has shown that the radar cross section, which is analogous to the dimensionless parameters $C_{i, k}$ and $D_{i, k}$ are a function of 
polarization and area of incidence. Since the transmitted waves were assumed to be vertically polarized, the area of incidence is the important factor in modelling these parameters. If the separation distance between the two mobiles is sufficiently greater than the distance between mobile and scattering object, the process of scattering at the transmitter and at the receiver may be assumed to be statistically independent. This is particularly the case if the receiver and transmitter are separated sufficiently far to approximate the sum of the waves travelling directly or via one or two reflections as a plane Transversal ElectroMagnetic wave through some plane perpendicular to the transmitterreceiver line of sight. This suggests $C_{i, k}=C_{i}$ and $D_{i, k}=D_{k}$. In the following analysis we will consider this to be a special case. Then, $E_{i, k}$ becomes equal to $C_{i} D_{k} E_{0}$, while $E_{i, 0}$ and $E_{0, k}$ tend to $C_{i} D_{0} E_{0}$ and $C_{0} D_{k} E_{0}$, respectively.

More in general, for reflections at both transmitter and receiver, the signal consists of a double sum over both reflections. If an unmodulated carrier is transmitted, the resulting electric field can be expressed as

$$
\begin{aligned}
& E(t)=E_{0,0} \cos \left[\left(\omega_{c}+\omega_{d}\right) t+\phi_{0,0}\right]+\sum_{i=1} E_{i, 0} \cos \left[\left(\omega_{c}+\omega_{T i}\right) t+\phi_{i, 0}\right] \\
& N_{R} N_{T} \\
& +\sum_{k=1} E_{0, k} \cos \left[\left(\omega_{c}+\omega_{R k}\right) t+\phi_{k}\right]+\sum_{k=1} \sum_{i=1} E_{i, k} \cos \left[\left(\omega_{c}+\omega_{R k}-\omega_{T i}\right) t+\phi_{i, k}\right]
\end{aligned}
$$

This field consists of a dominant component, which is treated deterministically, along with components that take into account reflections at the receiver, transmitter, and both receiver and transmitter. Measurements [15] indicated that at short range, both the line-of sight and the ground reflection are substantially stronger than the sum of weak scattered waves. Reflections off metal surfaces of the vehicle can also be strong. Hence, one may wish to model the channel using deterministic assumptions about strong paths, resulting in $E_{0,0}$, and using a statistical approach for $E_{i, 0}, E_{0, k}$ and $E_{i, k}$. In more detailed investigations, one can use the Rician channel model developed here but we will consider the dominant component $E_{0,0}$ to consist of the phasor sum of the line-of-sight and a ground reflection. 
The motion of the transmitter and receiver is evident in a Doppler shift in each wave component. Our model differs from the single reflection (Rayleigh fading) model by Akki and Haber [7]. However, for certain simplifying approximations both models lead to the same result (if the Rician $K$ factor of our model is chosen appropriately). From the geometry of Fig. 2.1, these Doppler shifts are found as follows:

$$
\begin{gathered}
\omega_{d}=\frac{2 \pi}{\lambda}\left(V_{R} \cos \gamma_{R}-V_{T} \cos \gamma_{T}\right) \\
\omega_{R k}=\frac{2 \pi}{\lambda} V_{R} \cos \left(\gamma_{R}-\alpha_{R k}\right) \\
\omega_{T i}=\frac{2 \pi}{\lambda} V_{T} \cos \left(\gamma_{T}-\alpha_{T i}\right)
\end{gathered}
$$

Here $V_{T}$ and $V_{R}$ are the velocities of the transmitter and receiver respectively and $\gamma_{T}$ and $\gamma_{R}$ are the angles that the motion of transmitter and receiver make with the road axis. In a typical IVHS environment vehicles are following each other, thus $\gamma_{T}=\gamma_{R}=0$. The received field can now be expressed as

$$
E(t)=I(t) \cos \omega_{c} t-Q(t) \sin \omega_{c} t+E_{0,0} \cos \left[\left(\omega_{c}+\omega_{d}\right) t+\phi_{0}\right]
$$

where

$$
\begin{aligned}
I(t)= & \sum_{i=1}^{\iota} E_{i, 0} \cos \left(\omega_{T i} t+\phi_{i}\right)+\sum_{k=1}^{\kappa} E_{0, k} \cos \left(\omega_{R k} t+\phi_{k}\right) \\
& +\sum_{k=1}^{N_{R}} \sum_{i=1}^{N_{T}} E_{i, k} \cos \left[\omega_{R k} t-\omega_{T i} t+\phi_{i, k}\right]
\end{aligned}
$$

and 


$$
\begin{gathered}
Q(t)=\sum_{i=1} E_{0,0} \sin \left(\omega_{T i} t+\phi_{i}\right)+\sum_{k=1} E_{0, k} \sin \left(\omega_{R k} t+\phi_{k}\right) \\
+\sum_{k=1} \sum_{i=1} E_{i, k} \sin \left[\omega_{R k} t-\omega_{T i} t+\phi_{i k}\right]
\end{gathered}
$$

If $N_{T}$ and $N_{R}$ are sufficiently large, in theory infinite (in practice Bennet [3] has shown that greater than 8 paths will suffice), the central limit theorem implies that both $I(t)$ and $Q(t)$ are Jointly Gaussian random variables for a particular time $t$ and the probability density of the angle of arrivals and departures is uniform between $(-\pi, \pi]$. If we assume that the separation distance between the two mobiles is much larger than the distance between the mobile and scattering object, then Clarke [1] has shown that both $I(t)$ and $Q(t)$ are uncorrelated and thus independent. The mean values of $I(t)$ and $Q(t)$ are both zero, the variance of $I(t)$ and $Q(t)$, or local-mean scattered power, is given by

$$
\sigma^{2}=\mathrm{E}\left[\sum_{i=1}^{N_{T}} \frac{E_{i, 0}^{2}}{2}+\sum_{k=1}^{N_{R}} \frac{E_{0, k}^{2}}{2}+\sum_{i=1}^{N_{T}} \sum_{k=1}^{N_{R}} \frac{E_{i, k}^{2}}{2}\right]
$$

and they are jointly Rayleigh distributed. Following Rice [6] we find that the joint probability density function of the received amplitude, $r(t)$, and phase, $\theta(t)$, is

$$
f_{r, \theta}(r, \theta)=\frac{r}{2 \pi \sigma^{2}} \exp \left(\frac{-\left(r^{2}-2 r E_{0,0} \cos \left(\theta-\omega_{d} t\right)+E_{0,0}^{2}\right)}{2 \sigma^{2}}\right)
$$

and thus the probability density function of the amplitude is given by

$$
f_{r}(r)=\frac{r}{\sigma^{2}} \exp \left(\frac{-\left(r^{2}+E_{0,0}^{2}\right)}{2 \sigma^{2}}\right) I_{0}\left(\frac{r E_{0,0}}{\sigma^{2}}\right)
$$

where $I_{0}($.$) is defined as the modified zero-order Bessel function of the first kind. We further define$ the Rician $K$ factor as the ratio of the power in the direct line-of-sight component to the local-mean scattered power 


$$
K=\frac{E_{0,0}^{2}}{2 \sigma^{2}}
$$

and define the local-mean power as

$$
\bar{p}=\frac{1}{2} E_{0,0}^{2}+\sigma^{2}=P_{D}+P_{R}+P_{T}+P_{B}
$$

where $P_{D}, P_{T}, P_{R}$ and $P_{B}$ are the portions of the local mean power in the dominant path, the waves that are scattered only near the transmitter, those scattered only near the receiver and those scattered twice, respectively. The pdf of the signal envelope $r$ can be expressed as

$$
f_{r}(r)=\underset{p}{=}(1+K) \exp \left(\frac{-K\left(1+K^{2}\right) r^{2}}{2 p}\right) I_{0}\left(r \sqrt{\frac{2 K 1+K}{\bar{p}}}\right)
$$

For the special case of sufficiently large antenna separation, we may further define Rician $K$ factors at the receiver and transmitter as the ratio of the power in the direct line-of-sight wave and the local-mean scattered power at the receiver and transmitter respectively, with

$$
K_{R}=\frac{D_{0}^{2}}{\mathrm{E}\left[\sum_{i=0}^{N_{R}} D_{i}^{2}\right]}
$$

and

$$
K_{T}=\frac{C_{0}^{2}}{\mathrm{E}\left[\sum_{i=0}^{N_{T}} C_{i}^{2}\right]} .
$$

The pdf of the signal envelope can be expressed in terms of these new Rician $K$ factors by making the following substitution of variables

$$
K=\frac{K_{R} K_{T}}{K_{T}+K_{R}+1+K_{R} K_{T}}
$$


We note that the resulting fading is Rician, which is similar to the case of a line-of-sight component with reflections occurring only at one of the antennas. Reflections at both transmitter and receiver are subject to two Doppler shifts. This results in a larger variance in both the in-phase and quadrature field components, which is tantamount to an increase in the scattered mean power.

\subsection{RF Spectrum}

The transmitted signal will be subject to Doppler shifts in the various paths. These Doppler shifts will tend to spread the bandwidth of the transmitted signal, which will be evident in the RF spectrum. The RF spectrum can be found by taking the Fourier transform of the temporal autocorrelation function of the electric field, the latter defined as

$$
\mathrm{E}[E(t) E(t+\tau)]
$$

Following Clarke [1], if we let

$$
a(\tau)=\mathrm{E}[I(t) I(t+\tau)]=\mathrm{E}[Q(t) Q(t+\tau)]
$$

and

$$
c(\tau)=\mathrm{E}[I(t) Q(t+\tau)]=-\mathrm{E}[Q(t) I(t+\tau)]
$$

then the autocorrelation can be expressed as

$$
\mathrm{E}[E(t) E(t+\tau)]=a(\tau) \cos \omega_{c} \tau-c(\tau) \sin \omega_{c} \tau+E_{0,0}^{2} \cos \left(\omega_{c}+\omega_{d}\right) \tau
$$

The parameters $C_{n}, D_{n}$ and $\phi_{\mathrm{n}}$ are statistically independent, due to the large separation distance between mobiles and the fact that small changes in path length will yield large changes in phase. Thus the following simplification can be made

$$
\begin{aligned}
& a(\tau)=P_{R} \mathrm{E}\left[\cos \omega_{R} \tau\right]+P_{T} \mathrm{E}\left[\cos \omega_{T} \tau\right]+P_{B} \mathrm{E}\left[\cos \left(\omega_{T} \tau-\omega_{R} \tau\right)\right] \\
& c(\tau)=P_{R} \mathrm{E}\left[\sin \omega_{R} \tau\right]+P_{T} \mathrm{E}\left[\sin \omega_{T} \tau\right]+P_{B} \mathrm{E}\left[\sin \left(\omega_{T} \tau-\omega_{R} \tau\right)\right]
\end{aligned}
$$

A critical assumption in [1] is that, for a large number of waves arriving at the receiver and departing at the transmitter, waves are modelled to arrive (or depart) from all angles in the azimuth plane with uniform probability density. For short range vehicle-to-vehicle communication this assumption is less obvious than for macro-cellular propagation environments. 
If, for ease of analysis, the probability density functions for $\alpha_{T}$ and $\alpha_{R}$ are nonetheless modelled by an independent uniform distribution between $(-\pi, \pi]$, we can now evaluate the above expectations as

$$
\begin{gathered}
a(\tau)=P_{R} \mathrm{~J}_{0}\left[2 \pi f_{M R} \tau\right]+P_{T} \mathrm{~J}_{0}\left[2 \pi f_{M T} \tau\right]+P_{B} \mathrm{~J}_{0}\left[2 \pi f_{M T} \tau\right] \mathrm{J}_{0}\left[2 \pi f_{M R} \tau\right] \\
c(\tau)=0
\end{gathered}
$$

where $\mathbf{J}_{0}($.$) is the zero-order Bessel function of the first kind and f_{M R}$ and $f_{M T}$ are the maximum Doppler shifts at the transmitter and receiver respectively given by

$$
f_{M T}=V_{T} / \lambda
$$

and

$$
f_{M R}=V_{R} / \lambda
$$

The fact that $c(\tau)$ is zero is a mathematical consequence of $\sin ($.$) being an odd function. Physi-$ cally this result can be related to the fact that the RF spectrum is symmetric about $f_{c}$. In order to calculate the power spectral density of $I(t)$ and $Q(t)$ we must first find the Fourier transform of $a(\tau)$. The Fourier transform of the first two terms can be found from Gradshteyn and Ryzhik [5,p.707] as

$$
\begin{gathered}
F\left\{P_{R} \mathrm{~J}_{0}\left[2 \pi f_{M R} \tau\right]+P_{T} \mathrm{~J}_{0}\left[2 \pi f_{M T} \tau\right]\right\}= \\
\frac{P_{R}}{2 \pi \sqrt{f_{M R}^{2}-f^{2}}} \Pi\left(\frac{f-f_{c}}{2 f_{M R}}\right)+\frac{P_{T}}{2 \pi \sqrt{f_{M T}^{2}-f^{2}}} \Pi\left(\frac{f-f_{c}}{2 f_{M T}}\right)
\end{gathered}
$$

where $\Pi(f / x)$ is the rectangular pulse function centered at $f=0$ with a width of $x$ and unity amplitude. The transform of the third term can be found [5, p.709] as 


$$
\begin{gathered}
F\left\{P_{B} \mathrm{~J}_{0}\left[2 \pi f_{M R} \tau\right] \mathrm{J}_{0}\left[2 \pi f_{M T} \tau\right]\right\}= \\
\frac{P_{B}}{2 \pi^{2} \sqrt{f_{M T} f_{M R}}} \mathrm{Q}_{-1 / 2}\left(\frac{f_{M R}^{2}+f_{M T}^{2}-f^{2}}{2 f_{M R} f_{M T}}\right) \Pi\left(\frac{f-f_{c}}{2 f_{M R}+2 f_{M T}}\right)
\end{gathered}
$$

where $\mathrm{Q}_{-1 / 2}($.$) is the Legendre function of the second kind. By using the following identity found [5]$

$$
\mathrm{Q}_{-1 / 2}(x)=\mathrm{K}\left(\sqrt{\frac{1+x}{2}}\right),
$$

the above transformation can be written in terms of $\mathrm{K}($.$) , the complete elliptical integral of the first$ kind, as

$$
\begin{gathered}
F\left\{P_{B} \mathrm{~J}_{0}\left[2 \pi f_{M R} \tau\right] \mathrm{J}_{0}\left[2 \pi f_{M T} \tau\right]\right\}= \\
\frac{P_{B}}{2 \pi^{2} \sqrt{f_{M T} f_{M R}}} \mathrm{~K}\left(\sqrt{\frac{\left(f_{M R}+f_{M T}\right)^{2}-f^{2}}{4 f_{M R} f_{M T}}}\right) \Pi\left(\frac{f-f_{c}}{2 f_{M R}+2 f_{M T}}\right)
\end{gathered}
$$

Setting $V_{T}=0$ we get an expression analogous to the expression in [1, p.969] for the baseband output spectrum from a square law detector. This output spectrum appears to be the convolution of the input spectrum with itself. This argument can be applied to our result. Namely the spectral contribution to the RF spectrum of the waves that undergo reflections at both receiver and transmitter, can be viewed as the convolution of the spectral components that undergo reflections only at the receiver with the spectral components that undergo reflection only at the transmitter. Stated mathematically,

$$
F\left\{P_{B} \mathrm{~J}_{0}\left[2 \pi f_{M R} \tau\right] \mathrm{J}_{0}\left[2 \pi f_{M T} \tau\right]\right\}=P_{B}\left\{F\left(\mathrm{~J}_{0}\left[2 \pi f_{M R} \tau\right]\right) \otimes F\left(\mathrm{~J}_{0}\left[2 \pi f_{M T} \tau\right]\right)\right\}
$$

The RF spectrum can now be found by noting that $a(\tau)$ is modulated by $\cos \omega_{c} \tau$, thus shifting the spectrum of $a(\tau)$ by the carrier frequency, and the direct line-of-sight wave will give rise to a delta function since this wave will only undergo a deterministic Doppler shift. Thus the RF spectra can be written as 


$$
S_{R F}(f)=F\left\{a(\tau) \cos \omega_{c} \tau+2 P_{B}\left[\cos \left(\omega_{c}+\omega_{d}\right) \tau\right]\right\}
$$

or

$$
\begin{aligned}
& S_{R F}(f)=\frac{P_{R}}{2 \pi \sqrt{f_{M R}^{2}-\left(f-f_{c}\right)^{2}}} \Pi\left(\frac{f-f_{c}}{2 f_{M R}}\right)+\frac{P_{T}}{2 \pi \sqrt{f_{M T}^{2}-\left(f-f_{c}\right)^{2}}} \Pi\left(\frac{f-f_{c}}{2 f_{M T}}\right) \\
& +\frac{P_{B}}{2 \pi^{2} \sqrt{f_{M T} f_{M R}}} \mathrm{~K}\left(\sqrt{\frac{\left(f_{M R}+f_{M T}\right)^{2}-\left(f-f_{c}\right)^{2}}{4 f_{M R} f_{M T}}}\right) \Pi\left(\frac{f-f_{c}}{2 f_{M R}+2 f_{M T}}\right)+P_{D} 2 \pi \delta\left(f-f_{c}-f_{M D}\right)
\end{aligned}
$$

where the Doppler shift of the direct line-of-sight component is

$$
f_{M D}=f_{M R} \cos \gamma_{R}+f_{M T} \cos \gamma_{T}
$$

In Fig. 5.2, we see that the RF spectrum is centered around the carrier frequency and bandlimited to $2\left(f_{M T}+f_{M R}\right)$ which is a direct consequence of the Doppler shift incurred by the movement of transmitter and receiver.

The probability densities of $\alpha_{R}$ and $\alpha_{T}$ affect the shape of the spectrum inside this band. If we set $V_{T}=0$ we do not obtain Clarke's spectrum for a mobile receiver and stationary transmitter. This is due to the fact that Clarke's model assumes no scattering at the transmitter. However if we set the Rician factors $K_{T}$ and $K_{R}$ to zero, we obtain a spectrum analogous to that of Akki and Haber [7] for a Rayleigh fading channel with scattering at transmitter and receiver only. 


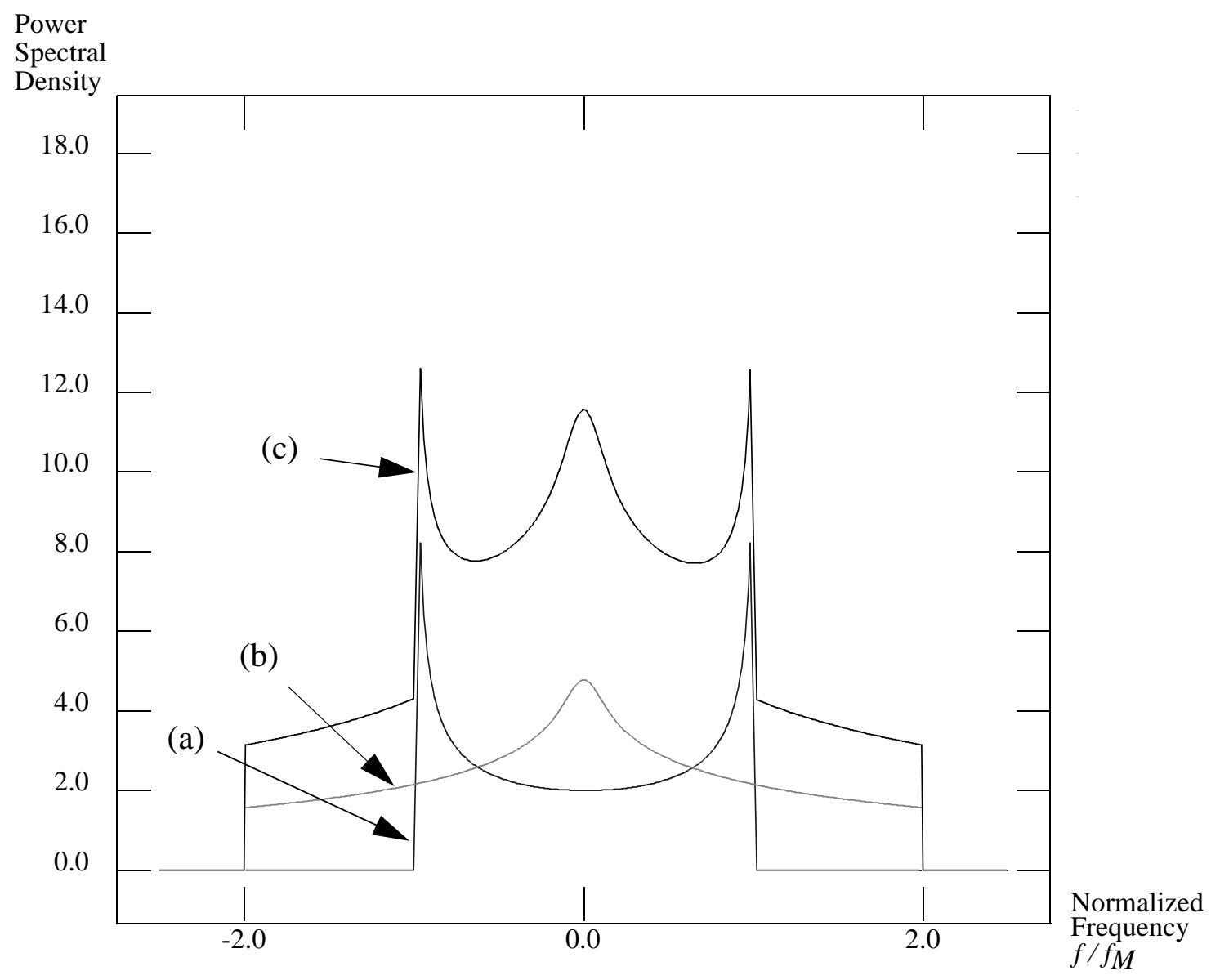

Figure 2.2 RF Spectra of mobile-to-mobile radio channel with equal transmitter and receiver velocities. (a) Reflections solely at either receiver or transmitter. (b) Reflections at both receiver and transmitter (c) summation of (a) and (b).

\subsection{Moments of Power Spectral Density}

The correlation functions $a(\tau)$ and $c(\tau)$ defined earlier can be expressed as inverse Fourier transforms of the power spectral density without the line-of-sight component as

$$
a(\tau)=\int_{f_{c}-\left(f_{M T}+f_{M R}\right)}^{f_{c}+\left(f_{M T}+f_{M R}\right)} S_{i}(f) \cos \left[2 \pi\left(f-f_{c}\right) \tau\right] d f
$$




$$
c(\tau)=\int_{f_{c}-\left(f_{M T}+f_{M R}\right)}^{f_{c}+\left(f_{M T}+f_{M R}\right)} S_{i}(f) \sin \left[2 \pi\left(f-f_{c}\right) \tau\right] d f
$$

where

$$
S_{i}(f)=S_{R F}(f)-E_{0,0}^{2} \pi \delta\left(f-f_{c}-f_{M D}\right)
$$

It was shown above that $c(t)$ is zero for all $t$ this can further be explained from the above equation since the RF spectrum is an even function while $\sin ($.$) is odd. These autocorrelations evaluated at$ zero will give expressions for the moments of the power spectrum. Following Jakes [4]

$$
\begin{aligned}
& \mathrm{E}\left[I^{2}(t)\right]=\mathrm{E}\left[Q^{2}(t)\right]=a(0)=b_{0}=P_{B}+P_{T}+P_{R} \\
& \mathrm{E}[I(t) Q(t)]=c(0)=0 \\
& \mathrm{E}[I(t) I(t)]=\mathrm{E}[Q(t) Q(t)]=\dot{a}(0)=0 \\
& \mathrm{E}[I(t) Q(t)]=-\mathrm{E}[I(t) Q(t)]=\dot{c}(0)=b_{1}=0 \\
& \mathrm{E}\left[I^{2}(t)\right]=\mathrm{E}\left[Q^{2}(t)\right]=-\ddot{a}(0)=b_{2} \\
& \qquad=\frac{1}{2}\left(P_{T} \omega_{M T}^{2}+P_{R} \omega_{M R}^{2}+P_{B}\left(\omega_{M T}+\omega_{M R}\right)^{2}\right)
\end{aligned}
$$

where the dots represent differentiation with respect to time. Thus $b_{n}=0$ for all odd $n$, again due to the symmetric nature of $S_{R F}(f)$. The moments of the power spectrum for even $n$ can be generalized as

$$
b_{n}=\left(\frac{1 \cdot 3 \cdot 5 \ldots n-1}{2 \cdot 4 \cdot 6 \ldots n}\right)\left(P_{T} \omega_{M T}^{n}+P_{R} \omega_{M R}^{n}+P_{B}\left(\omega_{M T}+\omega_{M R}\right)^{n}\right)
$$

Using the results of (37) we can now investigate the derivatives of the in-phase and quadrature components: specifically, to derive the joint pdf of these components and their derivatives. The inphase and quadrature components and their derivatives are zero-mean Jointly Gaussian. The covariance matrix can be expressed as 


$$
\boldsymbol{V}=\left[\begin{array}{cccc}
a(0) & c(0) & \dot{a}(0) & \dot{c}(0) \\
c(0) & a(0) & -\dot{c}(0) & \dot{a}(0) \\
\dot{a}(0) & -\dot{c}(0) & -\ddot{a}(0) & \ddot{c}(0) \\
\dot{c}(0) & \dot{a}(0) & \ddot{c}(0) & -\ddot{a}(0)
\end{array}\right]=\left[\begin{array}{cccc}
b_{0} & 0 & 0 & 0 \\
0 & b_{0} & 0 & 0 \\
& & & \\
0 & 0 & b_{2} & 0 \\
0 & 0 & 0 & b_{2}
\end{array}\right]
$$

so

$$
\boldsymbol{V}^{-1}=\left(\frac{1}{b_{0}^{2} b_{2}^{2}}\right)\left[\begin{array}{cccc}
b_{0} b_{2}^{2} & 0 & 0 & 0 \\
0 & b_{0} b_{2}^{2} & 0 & 0 \\
0 & 0 & b_{0}^{2} b_{2} & 0 \\
0 & 0 & 0 & b_{0}^{2} b_{2}
\end{array}\right] \text {. }
$$

The joint pdf [6],[10] can be written as

$$
f_{I, Q, I, Q}(I, Q, I, Q)=\frac{1}{4 \pi^{2} b_{0} b_{2}} \exp \left(\frac{-1}{2 b_{0} b_{2}}\left[b_{2}\left(I^{2}+Q^{2}\right)+b_{0}\left(I^{2}+Q^{2}\right)\right]\right) .
$$

The in-phase and quadrature components can be expressed in terms of an amplitude $r$ and phase $\theta$ as follows

$$
\begin{aligned}
& I(t)=r \cos \theta-E_{0,0} \cos \left(\omega_{d} t+\phi\right) \\
& Q(t)=r \sin \theta-E_{0,0} \sin \left(\omega_{d} t+\phi\right) \\
& I(t)=\dot{r} \cos \theta-r \dot{\theta} \sin \theta \\
& Q(t)=\dot{r} \sin \theta-r \dot{\theta} \cos \theta
\end{aligned}
$$

The joint pdf of the amplitude, phase and derivatives can be expressed as

$$
f_{r, \theta, \dot{r}, \dot{\theta}}(r, \theta, \dot{r}, \dot{\theta})=\frac{r^{2}}{4 \pi^{2} b_{0} b_{2}} \exp \left\{\begin{array}{c}
\frac{-1}{2 b_{0} b_{2}}\left[b_{2} r^{2}-2 r E_{0,0} \cos \left(\omega_{d} t+\theta\right)+E_{0,0}^{2}\right] \\
+b_{0}\left(\dot{r}^{2}+r^{2} \dot{\theta}^{2}\right)
\end{array}\right\}
$$


If we uncondition this expression over the phase and both derivatives, we obtain the same expression for the pdf of the signal envelope derived earlier (with $b_{0}=\sigma^{2}$ ).

\subsection{Level Crossing Rate and Average Fade Duration}

The fading of the signal envelope was evident in the derivation of the probability density function of the envelope. From this pdf we can obtain an expression for the overall percentage of time that the envelope lies below a certain level and on average how long these fades last. We are also interested in finding the rate at which the envelope crosses a particular level $R$. These expressions would thus provide parameters in selecting transmission bit rates, word lengths and coding schemes. The level crossing rate, $L_{R}$, is defined as the expected number of times per second that the envelope crosses $R$ in the positive direction. Rice [6] gives this value as

$$
L_{R}=\int_{0} \dot{r} f_{r, \dot{r}}(R, \dot{r}) d \dot{r}
$$

Thus we must first find the joint pdf of the envelope and its derivative. This can be derived by integrating the phase and its derivative over the joint pdf derived earlier.

$$
\begin{aligned}
f_{r, \dot{r}}(r, \dot{r}) & =\iint_{-\infty 0} f_{r, \theta, \dot{r}, \dot{\theta}}(r, \theta, \dot{r}, \dot{\theta}) d \theta d \dot{\theta} \\
& =\frac{r}{b_{0}} I_{0}\left(\frac{r E_{0,0}}{b_{0}}\right) \exp \left[\frac{-\left(r^{2}+\left(E_{0,0}\right)^{2}\right)}{2 b_{0}}\right] \times \frac{1}{\sqrt{2 \pi b_{2}}} \exp \left(\frac{-\dot{r}^{2}}{2 b_{2}}\right)
\end{aligned}
$$

From this expression we see that since both the envelope and its derivative are independent and thus uncorrelated, their joint pdf can be expressed as the product of individual pdf's. Thus the derivative of the envelope is zero-mean Gaussian with a variance of $b_{2}$ and the pdf of the envelope is the same as before. The level crossing rate then be expressed as

$$
L_{R}=\frac{R}{b_{0}} \sqrt{\frac{b_{2}}{2 \pi}} I_{0}\left(\frac{R \cdot E_{0,0}}{b_{0}}\right) \exp \left[\frac{-\left(R^{2}+E_{0,0}^{2}\right)}{2 b_{0}}\right]
$$


We further define the fade margin as the ratio of the mean signal power to the specified level, $R$. Fig. 2.3 plots the normalized level crossing rate, $L_{R} / f_{M}$ or level crossings per wavelength, for various Rician $K$ factors.

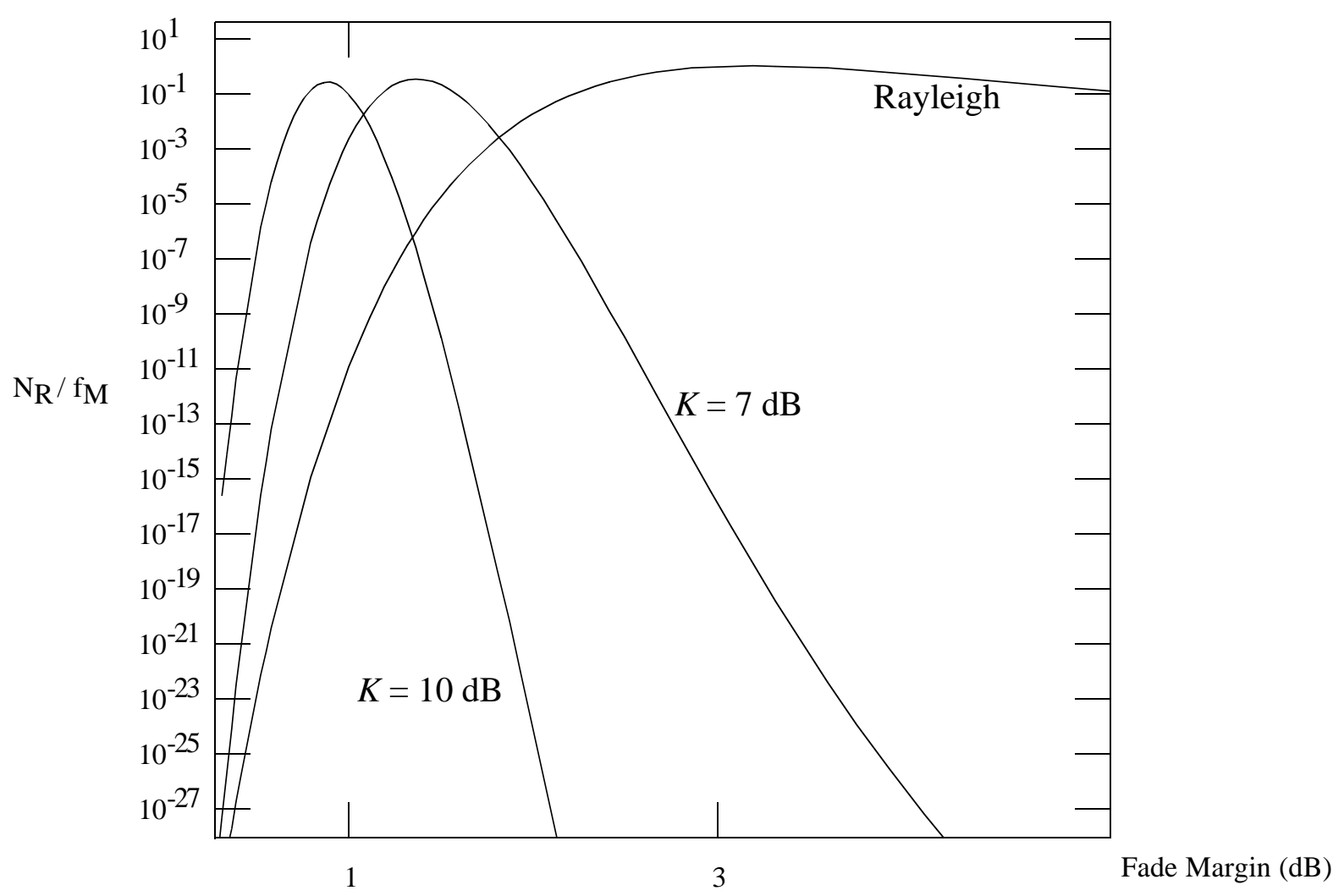

Figure 2.3 Normalized Level Crossing Rate vs. Fade Margin for various Rician $K$ factors. Equal transmitter and receiver velocities. Equal Rician $K$ factors $K_{R}$ and $K_{T}$.

Another important statistical measure of the envelope is the average fade duration. The fade duration, $\tau$, below a specified level $R$, is defined as the period of fade below this level. The overall fraction of time for which the signal is below a specified level $R$ is given by the cumulative distribution function, $F_{r}(R)$, of the received signal envelope. This function is obtained by integrating over the pdf of the envelope

$$
F_{r}(R)=\int_{0}^{R} f_{r}(r) d r
$$


The average fade duration can now be expressed as [9]

$$
E\left[\tau_{r}\right]=\frac{F_{r}(R)}{L_{R}}
$$

Fig. 2.4 plots the normalized average fade durations for various Rician $K$ factors.

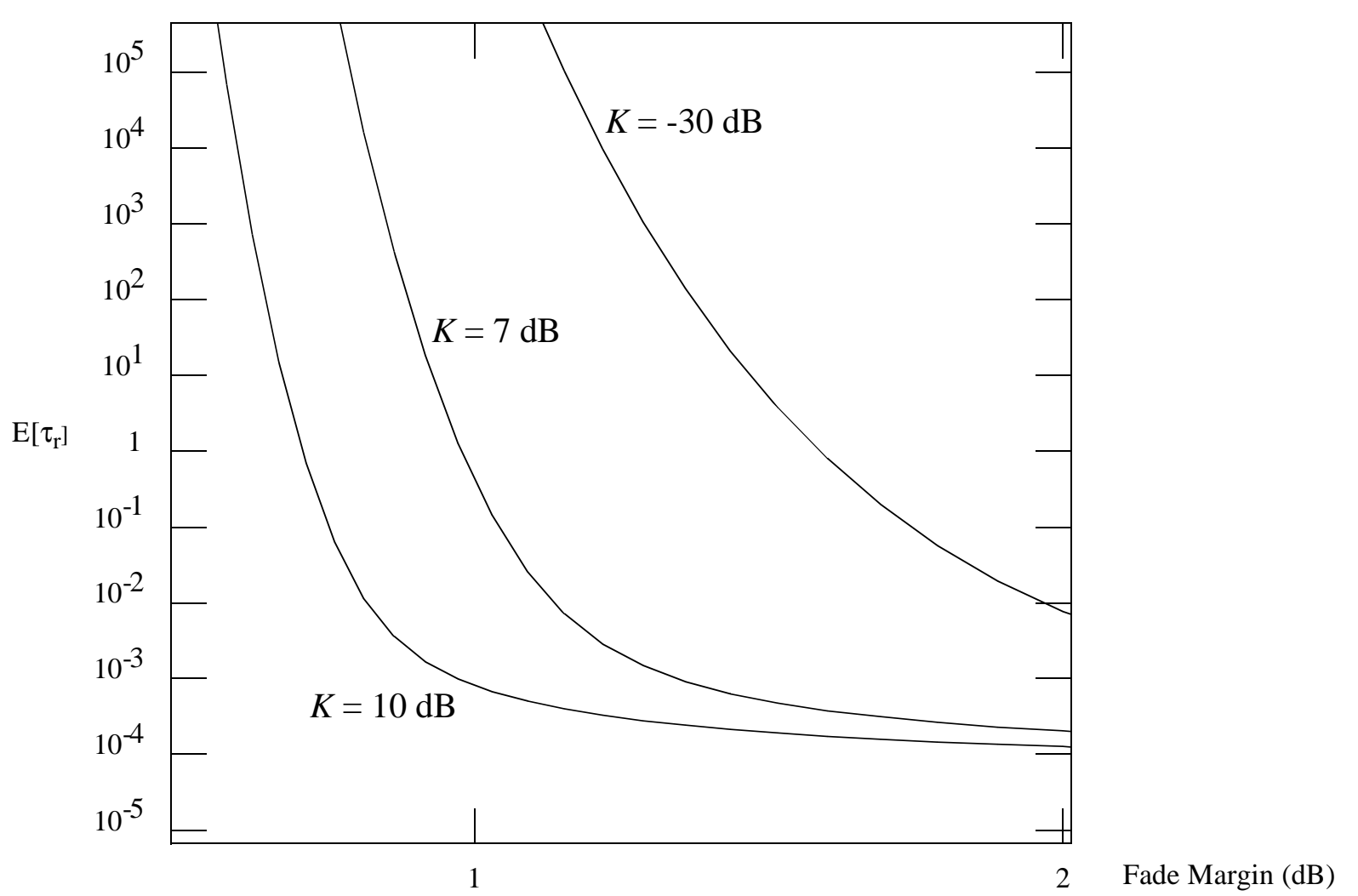

Figure 2.4 Average Fade Duration vs. Fade Margin for various $K$ factors. Equal transmitter and receiver velocities. Equal Rician $K$ factors $K_{R}$ and $K_{T}$. Maximum Doppler shift for both antennas, $f_{M}$

\subsection{Conclusions}

A statistical model for a mobile-to-mobile channel has been presented that extends the work on Rayleigh fading channels, without a line-of-sight component, by Clarke [1], Jakes [4], and Akki and Haber [7]. The channel model examines multipath fading and Doppler shifts for a narrowband signal 
taking into consideration scatters at both receiving and transmitting antennas both individually and concomitantly, as well as a strong line-of-sight component between antennas. This is in contrast to the model by Akki and Haber [7] which focussed on single reflections, ignoring a LOS or double reflections. The proposed model loses some of its accuracy when the transmit and receive antennas become too close. This suggests that the special case addressed here for independent scattering at the transmitter and receiver may be better acceptable for platoon-to-platoon communications, but needs further verification or it may need refinement for communication within a platoon.

Even with scattering at both transmitter and receiver, the line-of-sight component causes the fading to be Rician, with a new Rician $K$ factor that is a function of the $K$ factors at the transmitter, $K_{T}$, and receiver, $K_{R}$. As $K_{T}$ and $K_{R}$ approach zero we obtain results analogous to Akki and Haber [7] for Rayleigh fading with long range reflections. Also Doppler spreads, for vehicles travelling at roughly the same speed, are twice as large as those reported in text books like [4], thus the fading has components that are twice as fast. We interpret from our curves that fade durations longer than 50 msec diminish rapidly with fade margin. This implies that, if the fade margin is sufficiently large, the probability that the radio link is in an outage for longer than $50 \mathrm{msec}$ is very small. In an AVCS system, it appears important that longer outages become unlikely [14].

\subsection{References}

[1] R.H. Clarke, “A Statistical Theory of Mobile Radio Reception”, Bell. Syst. Tech. J., pp. 9571000, July 1968.

[2] A.L. Maffett, Topics for a Statistical Description of Radar Cross Section, New York: Wiley, 1989.

[3] W. R. Bennet, "Distribution of the Sum of Randomly Phased Components", Quart. Appl. Math., vol. 5, 1948, pp. 385-395.

[4] W.C. Jakes (Ed.), Microwave Mobile Communication, New York: Wiley, 1974.

[5] I.S. Gradshteyn and I.M. Ryzhik, Tables of Integrals, Series and Products, New York: Academic Press, 1965. 
[6] S.O. Rice, "Statistical properties of sine wave plus random noise,” Bell Syst. Tech. J., Jan. 1948, pp. 292-332.

[7] A.S. Akki and F. Haber, "A statistical model of mobile to mobile land communication channel," IEEE Trans. Veh. Technol.,vol. 35, no. 1, 1986, pp. 2-7.

[8] F. Bowman, Introduction to Bessel Functions, New York: Dover, 1958.

[9] D. Parsons, The Mobile Radio Propagation Channel, New York: Wiley, 1992.

[10] A. Leon-Garcia, Probability and Random Processes for Electrical Engineering, New York: Addison-Wesley, 1989.

[11] T. Aulin, "A modified model for the fading signal at a mobile radio channel," IEEE Trans. Veh. Technol., vol. 28, no. 3, 1979, pp. 182-203.

[12] W.B. Davenport and W.L. Root, An Introduction to the Theory of Random Signals and Noise, New York: McGraw-Hill, 1958.

[13] J.P.M.G. Linnartz, Narrowband Land-Mobile Radio Networks, Boston: Artech House, 1993.

[14] S. E. Shladover, et al., "Automatic vehicle control developments in the PATH Program," IEEE Trans. Veh. Technol, vol. 40, no. 1, Feb. 1991, pp. 114-130.

[15] J.S. Davis II and J.P.M.G. Linnartz, "Vehicle-to-vehicle RF propagation measurements", 28th Asilomar Conf. on Signals, Systems, and Computers, Pacific Grove, California, Nov. 1994, pp. 470-474. 
